\title{
Incorporación de contenidos de actividades en el medio natural y de ocio en la educación formal (TSEAS): Una experiencia de espeleología Incorporation of contents of activities in the natural environment and leisure in formal education (TSEAS): a caving experience \\ *Julio Fuentesal-García, ** David Zamorano-Sande *Universidad Pontificia Comillas (España), **Universidad Politécnica de Madrid (España)
}

\begin{abstract}
Resumen. El actual Real Decreto 653/2017, del 23 de junio que regula la formación de los técnicos superiores de enseñanza y animación socio deportiva ha abierto las puertas a nuevas intervenciones docentes. Como reflejo de su evolución en la educación y sociedad, las actividades en el medio natural han ganado relevancia dentro del currículum, invitando al alumnado a una inmersión en la naturaleza y aumentar experiencias de calidad al aire libre. En el presente artículo exponemos una intervención en progresión de dificultad de la espeleología realizada en el ciclo de TSEAS bajo este nuevo contexto curricular.
\end{abstract}

Palabras clave: actividades en el medio natural, espeleología, ocio, aventura, experiencia.

\begin{abstract}
The current Royal Decree-law 653/2017, of the 23 of June that regulates the formation of the superior grade formative course of animation and sports has encouraged new pedagogic interventions. As a reflection of its growth in society, the outdoor activities have increased their presence on the new curriculum, encouraging the implication of the students on outdoor activities and favoring quality experiences in the nature. On this article we present a innovative speleology intervention conducted with a group of superior grade formative course of animation and sports students, under the new curricular context.
\end{abstract}

Keywords: Outdoor activities, speleology, education, adventure, leisure, experience.

\section{Introducción}

Salir al campo es hoy en día una experiencia que rompe con nuestra rutina urbana, sin embargo antiguamente el medio natural era el escenario de nuestra vida (Aguila, 2007). Nuestra relación con el medio natural ha cambiado, nos hemos alejado de la relación que manteníamos en nuestros orígenes. Hoy en día se buscan aventuras imaginarias que hagan sentir emociones y sensaciones hedonistas fundamentalmente individuales, como forma de mantener la relación con el medio natural (Olivera \& Olivera, 1995).

Más allá de la evolución a un paradigma más individual y hedonista, se ha enfatizado la función de las actividades en el medio natural en diferentes entornos, como es el caso de la educación, autores como Blanco \& HernándezHernández (2017) y Granero-Gallegos (2010) han aportado su perspectiva a la dimensión educativa de estas actividades, haciendo énfasis en que las actividades en la naturaleza, tienen un fuerte vínculo con la educación (Granero-Gallegos, 2010).

Dentro de las actividades en el medio natural en la educación, uno de los vínculos más estrechos que existen con la educación formal es la educación física. Al llevar a cabo actividades en la naturaleza durante una clase de educación física, no todas las actividades guardan las mismas características, se diferencian en varios niveles de práctica (SantosPastor, 2003). Por un lado, se encuentra una actividad física general eminentemente educativa, que trata de favorecer el desarrollo integral del individuo a través del contacto e interacción con el medio, un ejemplo sería jugar al escondite. Por otro lado, una actividad específica y exclusiva del medio con intencionalidad educativa, como un paseo por el campo que se distingue de la anterior porque sólo puede desarro-

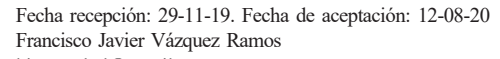

llarse en un entorno natural. Son estas últimas, las prácticas vinculadas necesariamente al medio natural, a las que haremos referencia en este documento, prácticas como la escalada, senderismo y espeleología (Escaravajal-Rodriguez \& Baena-Extremera, 2016; Fernandez-Río, 2009; Parra et al., 2008).

\section{Aplicación de las actividades del medio natural en la} educación formal.

Las actividades en el medio natural en educación física, se han vinculado a una variedad de posibilidades pedagógicas enriquecedoras, para Baena-Extremera (2011b) la naturaleza es el mayor aula de educación, ya que presentan unas posibilidades tan amplias que sobrepasan cualquier otro escenario educativo. Secuenciando algunos de estos beneficios destacamos:

Permitir trasladar la docencia a un ambiente saludable, algo cada vez más imperioso con el aumento de polución en las áreas urbanas (Granero-Gallegos \& Baena-Extremera, 2007).

Fomentar trabajar la creación de buenos hábitos en el estilo de vida, siendo un espacio propicio para el trabajo de sensibilización sobre la conservación del medio ambiente (Corrales, 2010). Esta sensibilización también abarca el equilibrio medioambiental entre las prácticas de actividades en medio natural y su impacto en el ecosistema (Sallent, 2009).

Por último, también se mejora la capacidad de adaptación en entornos desconocidos, desarrollando autonomía y auto-superación (Baena-Extremera et al., 2013).

A pesar de estos beneficios, la presencia de las actividades en el medio natural dentro de la educación formal se ha puesto en duda. Con respecto a ello, Sáez-Padilla et al. (2011) señalan la escasez de estas actividades en la educación secundaria, algo que según Tejada (2007) también ocurre en las primeras etapas educativas. La causa de esta ausencia de actividades se suele vincular a los conflictos a la hora de 
llevarlas a cabo dentro del currículum a impartir. Estas dificultades comienzan con que la formación del docente en estas áreas apunta a no ser muy abundante (Sáez-Padilla et al., 2017), teniendo una ínfima carga lectiva de asignaturas relacionadas con la naturaleza durante su formación (SaìezPadilla \& Fuentesal-García, 2014). Además, según PeñarrubiaLozano et al. (2011) el profesorado teme los riesgos que puede ocasionar estas prácticas al alumnado ( López et al., 2019), así como las consecuencias legales que pueden recaer sobre él, mientras ejerce y profesa como responsable de la actividad. Son muchos estudios e investigaciones los que apuntan a esta razón como una de las más determinantes a la hora de decidir la implantación de estas actividades, acrecentado por la poca cobertura jurídica que ampara al docente (Escaravajal-Rodriguez \& Baena-Extremera, 2016; SantosPastor \& Martinez-Muñoz, 2008).Otras dificultades serían la limitación horaria (Sáez-Padilla, 2008; Saìz-Padilla et al., 2011) teniendo en secundaría apenas dos horas de clase semanales para impartir estos contenidos. Esta limitación temporal guarda relación con la necesidad de desplazarse a las instalaciones o espacios donde desarrollar las actividades, habiendo muchos centros que carecen de dicha disponibilidad de tiempo, recursos y organización. Normalmente la organización de estas actividades en el medio natural recae en el docente, siendo este esfuerzo poco reconocido por el centro u otros compañeros. Además, muchas de las actividades suponen un coste añadido que no todos los centros están dispuestos a cubrir (Escaravajal-Rodriguez \& BaenaExtremera, 2016). Por último, y centrándonos no sólo en lo controlable por el ser humano, aparece el factor climático, éste puede alterar lo programado o incluso impedir su puesta en práctica.

Son muchas las limitaciones y beneficios que van de la mano de estas actividades y que se extienden en todas las etapas educativas (Granero-Gallegos et al., 2010). En la bibliografía existente se hacen continuas alusiones a las etapas de secundaria, primaria y educación superior, sin embargo, hay escasas referencias a los ciclos formativos de grado superior. Esta propuesta se contextualiza en el ciclo superior de Técnico superior en enseñanza y animación socio deportiva (en adelante TSEAS).

\section{Contextualización de la propuesta}

Recientemente ha entrado en vigor el Real Decreto (en adelante RD) 653/2017, del 23 de junio a través del cual se regula la titulación del TSEAS. Este RD deroga al RD 1262/ 1997, de 24 de julio, por el que se establecía el currículo del ciclo formativo de grado superior correspondiente al título de Técnico superior en animación de actividades físicas y deportivas (en adelante TAFAD). No debemos confundir del TSEAS con el Técnico en conducción de actividades físico-deportivas en el medio natural, (en adelante TECO). Tampoco se debe confundir con la formación de los Técnicos deportivos, ya que al igual que el grado TECO, tienen unas competencias profesionales divergentes a las de un TSEAS, las cuales se abordan de forma superficial más adelante en el documento. De nuevo con TSEAS, durante los veinte años de vigencia del decreto derogado mencionado previamente, se han desarrollado nuevas disciplinas depor- tivas y formas de entender la enseñanza deportiva como es el caso de las actividades físicas en el medio natural. Este incipiente cambio de paradigma en la enseñanza deportiva ha hecho cada vez más evidente la necesidad de un nuevo currículum que de cabida a estas nuevas prácticas. Para dar respuesta a esta demanda, en el vigente $\mathrm{RD}$ aparecen los nuevos módulos profesionales a impartir, entre ellos el de actividades de ocio y tiempo libre, el que más relación guarda con las actividades físicas en el medio natural.

Esta variación con respecto al anterior decreto, hace explícita la presencia de las actividades físicas en el medio natural dentro del currículum a impartir. La impartición de contenidos como el de esta propuesta se justifica en las competencias profesionales que recoge la legislación vigente. Estas competencias hacen referencia a la capacidad de programar, diseñar y adaptar actividades, recreaciones y juegos en varios contextos y adaptándolas a los participantes, competencias que adquieren a través de las experiencias y aprendizajes de estas prácticas. No obstante, aunque esos contenidos guarden relación con las competencias profesionales, estén respaldados por la legislación y exista más libertad a los docentes a la hora de llevar a cabo la impartición de su currículum, la redacción de los criterios deja a los docentes en la tesitura de elegir los contenidos para alcanzar los resultados de aprendizaje estipulados (Sáez-Padilla et al., 2017).

Esto provoca indirectamente que la realización de actividades en la naturaleza resida en la implicación del docente, a veces relacionada con su formación en las actividades en el medio natural. La formación de los docentes en estas actividades, como ya hemos abordado en este artículo, es insuficiente (Sáez-Padilla \& Fuentesal-García, 2014). Aún teniendo el conocimiento, los docentes no abordan ciertos contenidos por el tiempo de organización, limitaciones espaciales, temporales, cobertura jurídica, riesgos que ocasiona, reconocimiento de agentes educativos, entre otras (EscaravajalRodriguez \& Baena-Extremera, 2016; Santos-Pastor \& Martinez-Muñoz, 2008).Como alternativa a estas adversidades algunos docentes, optan por la colaboración con técnicos especialistas con una formación más específica para que se encargue de impartir estas actividades más técnicas (SáezPadilla et al., 2017). En el caso de nuestra propuesta a pesar de que el docente tuviese conocimientos de las técnicas de progresión vertical, escalada y cacharrería, decidió delegar la responsabilidad de la actividad, a la Federación Madrileña de Espeleología (en adelante FME). La FME es la máxima representación de la disciplina en la Comunidad de Madrid. Dentro de las funciones que ejerce está la de promover actividades de formación, tecnificación y divulgación de la espeleología, esta última función es la que la hace participe de actividades como la presentada en este artículo. A través de la divulgación de la espeleología en centros educativos la FME facilita el acercamiento de esta disciplina al alumnado. El papel de las Federaciones es crucial para la expansión de estos deportes menos reconocidos y son una pieza clave para la propagación de actividades minoritarias. Así, tanto de las federaciones como desde el contexto escolar podemos influir para que el alumnado aumente sus niveles de práctica deportiva intentando conectar el contexto académico con el del ocio para transferir conocimientos, destrezas y 
actitudes que el discente pueda mantener a lo largo de su vida. No obstante, estas iniciativas deben ampararse y ajustarse siempre a cada etapa educativa, como se ha realizado con la presente propuesta (Ferriz et al., 2018).

El contenido que se ha decidido abordar en el modulo de actividades de ocio y tiempo libre dentro de este artículo es la espeleología. La propuesta se enmarca en la literatura existente dentro de otras experiencias de acercamientos a la espeleología (Baena-Extremera \& Granero-Gallegos, 2009; Escaravajal-Rodriguez \& Baena-Extremera, 2016). Contribuye en lo ya investigado, expandiendo lo ya existente a través de incorporar una nueva experiencia en el ámbito real de práctica y con discentes pertenecientes a TSEAS. La espeleología ofrece a este perfil profesional herramientas para su futura responsabilidad como monitores de actividades en el medio natural, éstas están alineadas con las competencias profesionales previamente mencionadas. Además, contextualizar la experiencia en TSEAS ha permitido explorar las posibilidades dentro de la nueva legislación, aportando a la escasa literatura existente que aborda este perfil profesional.

\section{Introducción de la propuesta}

Durante la evolución de la espeleología, ésta ha sido definida por muchos autores en la sociedad (AA.VV, 2007; Diamant, 1991). Para no extendernos en el texto seleccionamos a Baena-Extremera (2008) que la define como la progresión en cavidades subterráneas mediante el empleo de los métodos y materiales característicos de la escalada y de las técnicas verticales.

Las singularidades inherentes a la espeleología hacen que sea una actividad estimulante a la hora de acercarla al aula como hemos visto en otras propuestas (Baena-Extremera \& Granero-Gallegos, 2009; Escaravajal-Rodriguez \& BaenaExtremera, 2016). El interés y necesidad de inyectar en el alumnado nuevas dosis de motivación, hace que el mundo docente adapte, actualice y acerque innovadoras propuestas en sus clases saliéndose de lo habitual y rutinario (Fuentesal-García \& Pastor, 2019).

En este tipo de propuestas prácticas, la superación y confianza en uno mismo pone en juego el desarrollo de habilidades y competencias personales sin necesidad de un oponente directo, una de las metas que se persigue, es simplemente salir victorioso de ella (Gómez-Encinas, 2008).

Teniendo en cuenta estas características de la espeleología, el establecimiento del nuevo currículum y la alineación con las competencias profesionales, se ha decidido dirigir la presente propuesta a las enseñanzas deportivas de TSEAS. Aunque también se podría adaptar a otros ciclos como primaria, secundaria o el ámbito universitario. El objetivo de la práctica es aproximar al alumnado a la iniciación en la espeleología a la vez que da a conocer al profesorado perspectivas sobre cómo trabajar otros deportes de aventura emergentes, ya puede ser espeleología u otras disciplinas como escalada, buceo, ciclismo, etc... Pudiendo complementarse con otras propuestas educativas similares a la que presentamos (Aguilar \& Machota, 2014; Gómez \& Sanz, 2003; Navarro, 2018; M. Sanjuán et al., 2020).

\section{Presentación de la propuesta}

Este trabajo se encuadra en el módulo profesional de actividades de ocio y tiempo libre del ciclo de TSEAS, dónde tendremos en cuenta y tomaremos de referencia el RD 653/ 2017, de 23 de junio por el que se establece dicho título. Con la presentación de nuestra propuesta intentamos poder alcanzar y contribuir a que nuestro alumnado logre el mayor número de objetivos propuestos, así como resultados de aprendizaje. Para no extendernos nombraremos uno de los más relacionados y prioritarios en nuestro trabajo, como es el de realizar actividades de ocio y tiempo libre en el medio natural, respetando los principios de conservación del medioambiente y las medidas de prevención y seguridad (García, 2002).

Estas actividades podrían considerarse como una fórmula perfecta para potenciar el desarrollo sostenible, en ocasiones la ausencia de legislación que asegure la conservación de estos espacios hace aún más importante la labor del profesorado e instituciones como la FME para concienciar a la población de los riesgos y repercusión medioambiental de la práctica de estos deportes. Por ello, colocaremos la lupa a lo largo de nuestras sesiones para reflexionar y hacer reflexionar al alumnado sobre la importancia de estos conceptos modificando nuestro ambiente de aprendizaje trasladándonos al aire libre.

A continuación, mostramos las seis sesiones de nuestra propuesta, en ellas tendremos en cuenta la progresión de dificultad que hay que plantear en este tipo de deportes, y tomaremos referencia de otros trabajos que se han abordado anteriormente (Baena-Extremera et al., 2013; Cano et al., 2009).

Recordamos que la propuesta esta contextualizada en el $2^{\circ}$ Ciclo de TSEAS durante el segundo trimestre de una clase de veinticuatro alumnos/as en Madrid capital.

\section{Objetivos}

- Descubrir la disciplina de la espeleología como deporte-ciencia.

- Conocer el medio donde se desarrolla la práctica de la espeleología.

- Usar correctamente los materiales de la espeleología.

- Ejecutar correctamente las técnicas básicas de espeleología.

- Distinguir las características básicas del entorno subterráneo.

- Identificar los accidentes geográficos de una cueva.

- Respetar las normas de seguridad.

- Aprender el protocolo en caso de accidente o necesidad de operativo de rescate.

- Identificar las necesidades organizativas como futuro responsable de este tipo de actividades.

\section{Contenidos}

- Normas de seguridad y prevención de riesgos en las actividades del medio natural.

- Utilización de materiales de progresión específicos de la espeleología.

- Técnicas de progresión por pasamanos, descenso y 
ascenso básico.

- Código de comunicación.

- Técnicas de descenso y rapel básico.

- Morfología de las cuevas y características autóctonas.

- Diagnóstico, planificación, ejecución y evaluación de una actividad en el medio natural.

- Responsabilidad, protocolos, riesgos y cobertura legal en la organización de actividades al aire libre.

- Legislación local.

\section{Criterios de evaluación}

A continuación, hemos vinculado los criterios recogidos del RD 653/2017 con las acciones llevadas a cabo durante la actividad, para ilustrar cómo se han alcanzado:

- Se han aplicado técnicas y herramientas coherentes con los objetivos del proyecto

de actividades de ocio y tiempo libre en el medio natural: conocimiento de la disciplina de la espeleología, de la mano de la FME. Descripción de las características y herramientas necesarias para el desarrollo de una progresión en cueva.

- Se han seleccionado estrategias y pautas de actuación para el desarrollo de

actividades educativas en el medio natural: participación en la preparación de la actividad en la cueva, implicando el conocimiento de las características de la actividad, así como las medidas de seguridad y técnicas para la progresión en la cueva.

- Se ha valorado la importancia de generar entornos seguros en el medio natural,

minimizando riesgos: consciencia de la progresión en dificultad y seguridad física y psíquica de los discentes durante los ejercicios de rapel y cabuyería con visión a la aplicación técnica en un entorno real.

- Se han identificado los recursos necesarios para el desarrollo de las actividades

en el medio natural: conocimiento del material necesario para la progresión en las cuevas y técnicas de rappel, formación de técnicos e instalaciones.

- Se han organizado los recursos humanos para garantizar el desarrollo de la

actividad: organización del grupo, dinamización en la salida al medio natural, así como reconocer las necesidades de un grupo en el desarrollo de la actividad.

- Se han aplicado protocolos de atención en casos de situaciones de emergencia en

las actividades desarrolladas en el medio natural: conocimiento de los protocolos y las medidas a tomar en caso de presentarse situaciones de emergencia dentro de una cueva: botiquín en la puerta de la cueva, utilización de emisora, control activo del grupo y cálculo del tiempo de la actividad.

\section{Metodología}

Este apartado lo hemos querido abordar tomando como referencia la propuesta de Baena-Extremera (2011b) en la que diferencia dos partes: una experimental y otra práctica. Por lo tanto, la parte experimental serían las sesiones en el centro y la parte práctica la actividad en la cueva de la bruja.

La sección experimental se subdivide en dos a su vez, durante las primeras sesiones en el centro, donde nos encontrábamos en las primeras fases de familiarización y asentamiento de conceptos básicos relacionados con el control del material, iniciación a la cabuyería y disciplina. Se ha utilizado la asignación de tareas, según la clasificación de Delgado (Hernández, 2008).

En las sesiones posteriores aún en el centro, una vez asentados estos conocimientos básicos, se utilizó la micro enseñanza lo que permite que por medio de una práctica simulada se resuelvan situaciones reales tal y como se van a presentar en el medio real (León \& Santiago, 2014).

\section{Instalaciones y material}

- Instalaciones: el propio pabellón, columnas, espalderas, escalera suspendida, rellano con desplome y zona de rocódromo.

- Instalaciones fuera del centro: cueva de la bruja situada en Alpedrete de la Sierra. Es una cueva de tres pisos con una entrada de unas pequeñas dimensiones que se ensancha en una galería. Las columnas permiten establecer anillos de cuerda para realizar el rappel de descenso de unos treinta metros. Es una cueva de una dificultad media-baja.

- Material móvil: cordinos, arneses, mosquetones de aluminio, mosquetones de acero, cascos de escalada, cascos de espeleología, frontales, cuerdas, colchonetas, stop, ochos, gri.gri, monos de espeleología y botiquín de primeros auxilios.

- Organización del grupo: en las sesiones llevadas a cabo en el centro, la ratio era de un profesor por cada nueve alumnos/as. En la práctica externa la ratio era de un técnico por cada ocho discentes más el docente del centro y el profesor de prácticas.

- Docentes: durante las sesiones en el centro, dirigieron la sesión un docente acompañado de un profesor en prácticas. La sesión que dio el técnico de espeleología en el centro fue dirigida por él en solitario, acompañado del docente habitual de la asignatura y el profesor en prácticas. La salida fuera del centro estuvo coordinada enteramente por dos técnicos de la FME acompañados por el docente que se quedó fuera de la cueva y el docente en prácticas. Los dos técnicos y el profesor en prácticas entraron en la cueva con el alumnado y se comunicaban con el docente a través de una emisora de forma regular para comunicar el avance de la situación.

\section{Desarrollo de la experiencia de espeleología.}

A continuación, se desarrolla la propuesta de espeleología aplicando la metodología, material, contenido y objetivos presentados anteriormente. Distinguimos las siguientes fases: fase experimental y conocimiento compuesta por cinco sesiones y fase práctica compuesta por una sesión.

\section{Fase experimental y de conocimiento}

\section{$1^{\circ}$ Sesión: Iniciación a la espeleología y educación} ambiental.

En la primera sesión se abordarán tres grandes bloques: 
introducción, concienciación y planificación. La introducción a la espeleología se va a basar en exponer conocimientos teóricos básicos que caracterizan la disciplina. En segundo lugar, debido al contexto donde se realiza la práctica, es necesario hacer hincapié en el impacto medioambiental, sus consecuencias y forma de prevenirlo (Tejada \& Sáez-Padilla, 2009). Por último, se presenta al alumnado la planificación de la intervención, detallando las medidas de seguridad, progresiones y representaciones de la cueva donde se realizará la última de nuestras sesiones.

Esta sesión se lleva a cabo en un aula convencional equipada con un proyector apoyándonos en el visionado de videos e imágenes para representar los contenidos abordados. (imagen 1)

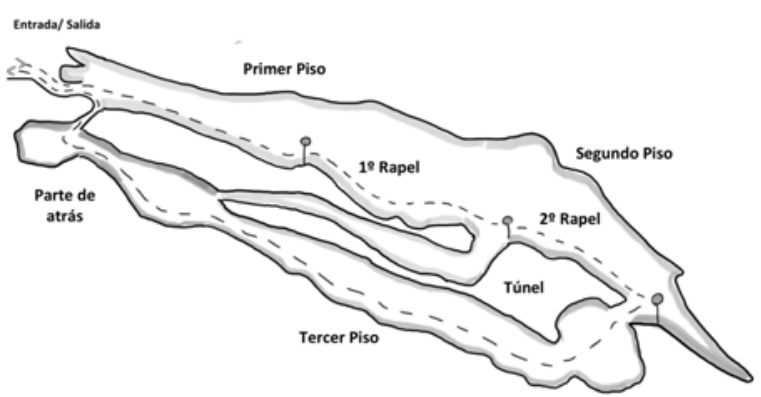

Imagen 1. Croquis de la cueva de la Bruja. Fuente: David Zamorano, CES Don Bosco (Madrid), 2020.

\section{$2^{\circ}$ Sesión: Cabuyería y muestra del material}

En la segunda sesión los alumnos/as tienen un primer acercamiento a los materiales para comprender su función en la actividad.

Durante la sesión se presenta a los discentes la cabuyería básica que van a necesitar, los nudos que se van a impartir, nudos que tienen transferencia a otras actividades cómo la escalada: el ocho, ocho cosido, ocho con doble gaza y as de guía como fundamentales (Navarro, 2018). También se presenta el material básico que se va a utilizar: cuerdas semiestáticas, mosquetones de seguridad, cascos de escalada y arneses. Una vez conocido lo básico, se introducen los materiales de rapel como el ocho y el stop. Por último, se habla del rol organizador de la actividad para que los alumnos/as, futuros organizadores sean partícipes de los trámites e información necesaria para su realización. En base a esto, se habla de los casos de prevención y rescate, las actividades paralelas y por último el aforo de la cueva y cómo esta ratio es determinante para su preservación. Respecto a la actividad paralela, mientras que los alumnos/as del primer

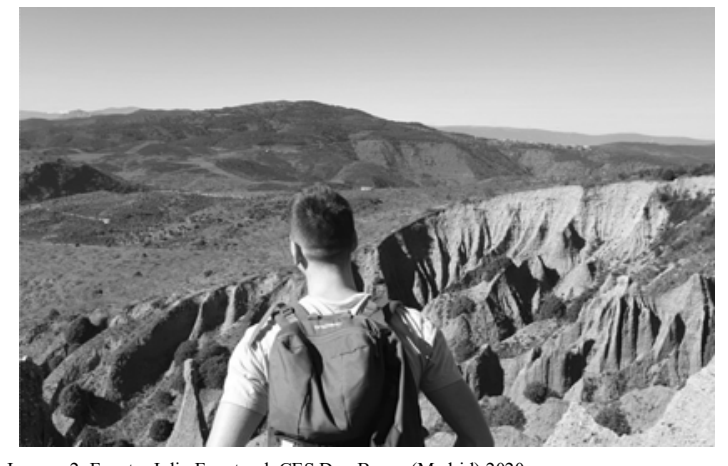

Imagen 2. Fuente: Julio Fuentesal, CES Don Bosco (Madrid),2020 turno realizan la cueva, los discentes del segundo turno realizan una actividad de sendero guiado visitando la zona de las Cárcavas. (imagen 2)

Esta sesión se lleva a cabo en un aula convencional equipada con un proyector, apoyándonos en el visionado de videos para ejemplificar el uso de los materiales en la práctica real y mostrar ejemplos de uso de la cabuyería.

\section{$3^{\circ}$ Sesión: Realización técnicas de rappel}

Para empezar esta sesión se recuerda el material, haciendo hincapié en el que se va a utilizar durante la práctica de la sesión. Es fundamental repasar la colocación del arnés y sus partes así como el funcionamiento del ocho (Navarro, 2018).

Una vez probado el equipamiento y obtenida cierta confianza, el profesor facilitará al alumnado el acercamiento a la práctica. Las características de la cueva hacen que no sea necesario utilizar técnicas de ascensión con cuerda, la cueva tiene galerías que permiten transitarla con o sin rapel. Por lo tanto, nos centraremos en las técnicas básicas de rapel con ocho. Para ello se comenzará con progresiones horizontales y más adelante incrementar la dificultad con progresiones verticales (Escaravajal-Rodriguez \& Baena-Extremera, 2016).

A nivel organizativo, cada alumno/a dispondráì de su material en la zona de espera, cuando sea su turno y habiendo sido revisado el material por un compañero y finalmente por el profesor, procederá a rapelar. El discente tendrá el ocho en su anillo ventral, mientras que el compañero y profesor aseguran agarrando la cuerda que proviene de su ocho. Tras cada rapel, el alumno/a debe esperar su turno para poder comenzar el siguiente. Estas son las dos estaciones de progresión realizadas en esta primera sesión.

Rappel en horizontal al suelo: Estando el alumno y el docente en bipedestación, siendo el compañero y el docente el que asegura al alumno, se establece un punto de anclaje en la columna. El alumno simula el movimiento de rapelar desplazándose hacia atrás, mientras le aseguran.

Rapel en una superficie con leve inclinación: Se establece un punto de anclaje en lo más alto de un plano inclinado de cuarenta y cinco grados, formado por varios bancos suecos apoyados en las escaleras. El compañero y el profesor aseguran al alumno que simula los desplazamientos de un rappel descendiendo en el plano inclinado.

Para finalizar se recoge el material y se pide un feedback del alumnado respecto a las sensaciones durante la actividad. Esta práctica se lleva a cabo en el pabellón del centro con el material necesario para realizar la técnica de rapel.

\section{$4^{\circ}$ Sesión: Rappel vertical y volado}

La sesión comienza con un repaso de las técnicas vistas durante la sesión anterior, de cara a volverlas a aplicar, en situaciones de mayor dificultad. Siendo el objetivo que el alumnado aprenda las técnicas y movimientos necesarios para rapelar y progresar por una cueva, durante esta sesión se modifica la organización para cumplir con ese objetivo. Utilizando el estilo de microenseñanza (Solana \& Delgado, 2005), será el alumnado el que transmita a sus compañeros las técnicas fomentando que se corrijan entre ellos. En este momento, los alumnos/as rapelan con el ocho mientras que otro discente les asegura agarrando la cuerda que proviene de su ocho, de esta forma el alumno/a toma consciencia de 
su destreza en las maniobras. Es en este momento cuando el discente emite un juicio de sus conocimientos y el de sus compañeros para consecuentemente tomar decisiones autónomas que afecten a su aprendizaje (Fraile et al., 2020). Este proceso se realiza en cinco estaciones simultaneas, para obtener fluidez y que el tiempo de compromiso motriz sea mayor en cada alumno/a (Martínez et al., 2007; Muñoz et al., 2017). El docente adopta la función de supervisor, interviniendo para enfatizar en la seguridad de la actividad en todo momento.

Al explicar al alumnado la estructura organizativa, se comienza la actividad. Todas las estaciones de progresión se realizaron en la primera sesión y se repitieron en la segunda $\mathrm{y}$ fueron continuadas por estas tres estaciones.

Formación en aseguramiento con cuerda auxiliar: En la parte superior de la instalación, se colocará una cuerda independiente a la de progresión para trabajar el aseguramiento con cuerda independiente mediante nudo dinámico dando al alumnado dos herramientas de aseguramiento.

Rapel con apoyo de pies: En un desnivel plano, formado por las escaleras del gimnasio, se realiza un punto de anclaje desde el cual el alumnado rapela y llega a una colchoneta a tan solo tres metros de distancia.

Rapel volado: Aprovechando las escaleras ancladas en paralelo al suelo del gimnasio, se realiza un punto de anclaje donde el alumnado realiza un rapel suspendido, sin apoyo de pies en una distancia de tres metros de alto.

Es importante destacar que a pesar de que estas progresiones se realicen rapelando con el ocho, en la práctica real se utiliza el stop. Consideramos que manejando el ocho existe una transferencia positiva a la utilización del stop, siendo más sencillo el manejo del stop, porque realiza bloqueo automático de la cuerda sin necesidad de realizar ninguna acción sobre él.

Esta práctica se lleva a cabo en el pabellón del centro y en el rocódromo apoyándonos en ambos espacios para rea-

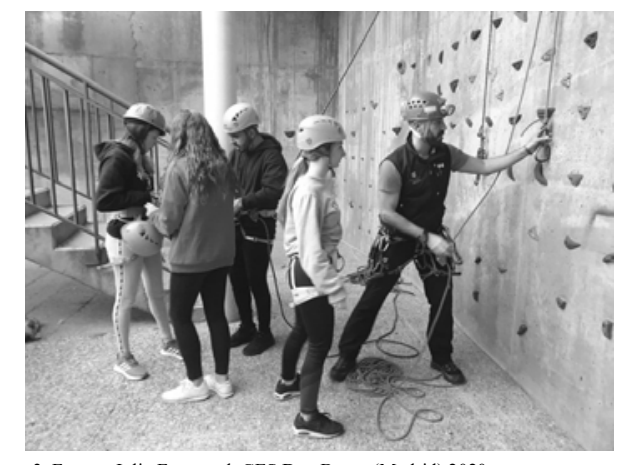

Imagen 3. Fuente: Julio Fuentesal, CES Don Bosco (Madrid),2020

lizar las técnicas más verticales. (imagen 3)

\section{$5^{\circ}$ Sesión: Visita FME}

En esta sesión un técnico de la FME vino al centro a impartir una sesión puente entre la práctica en el pabellón y la cueva. (imagen 4)

Esta sesión consistió en una clase magistral que siguió este esquema de contenidos:

- Morfología de las cuevas: Peculiaridades de la morfología subterránea, normas para su preservación, protocolos y características de la zona.

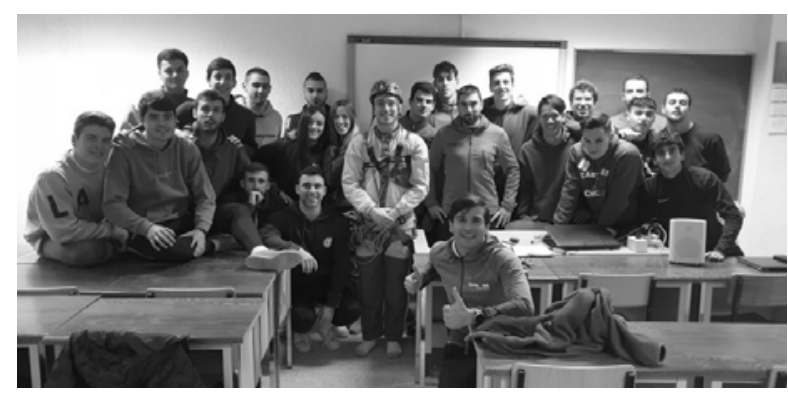

Imagen 4. Fuente: Julio Fuentesal, CES Don Bosco (Madrid),2020

- Organización de una actividad: Trámites burocráticos, permisos, seguros a gestionar como organizador de una actividad en el medio natural, división de los grupos, normas a cumplir durante la actividad, material obligatorio y recomendaciones de alimentación e hidratación.

- Presentación del material específico: Casco de espeleología, mono, arnés y stop. (imagen5)

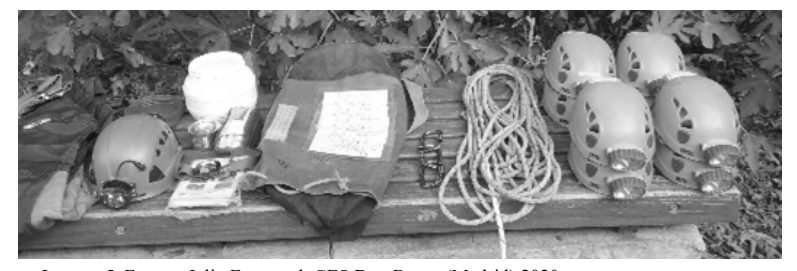

Imagen 5. Fuente: Julio Fuentesal, CES Don Bosco (Madrid),2020

\section{Fase práctica}

\section{$6^{\circ}$ Sesión: Experiencia en la cueva}

En esta fase, siguiendo con la progresión propuesta por Rodríguez \& Baena-Extremera (2016) se culmina la experiencia con una actividad en el medio real. Para ello se desplazó al grupo de alumnos/as a Patones, un municipio al noreste de Madrid perteneciente a la provincia de dicho municipio. En Patones, realizamos el descenso a la Cueva de la Bruja. Toda la actividad estuvo coordinada por la FME. Los dos técnicos de la federación realizaron un briefing previo a la entrada en la cueva, recordando los elementos más importantes del material y asegurando que todo el alumnado estaba al corriente de ello.

Durante el descenso de la cueva, que tuvo una duración aproximada de tres horas, hubo una comunicación constante con el grupo. En las galerías más amplias se aprovechó para ver in situ las características morfológicas de las cuevas que se habían abordado en la anterior sesión. El descenso de la cueva estuvo marcado por dos vías de rapel de aproximadamente diez metros cada una, donde el alumnado se autorapelaba con un stop guiado por los técnicos. En este momento los alumnos/as pusieron en práctica las técnicas abordadas en las progresiones propuestas en el inicio de la experiencia. Hay que resaltar que en todo momento los técnicos prestaron especial atención al alumnado que había mostrado cierta inquietud o nerviosismo al comenzar la actividad, para ello hicieron hincapié sobre las diferentes vías de escape y se les realizó un acompañamiento más cercano.

En relación con las normas de seguridad, los técnicos habían realizado la cueva en otras muchas ocasiones y estaban equipados con material suficiente y botiquín de primeros auxilios. Un docente del centro se quedó fuera de la 
cueva permitiendo establecer un enlace con el exterior que estimaba el tiempo de duración de la actividad y podía avisar a los servicios de emergencia en caso de un posible imprevisto.(imagen 6)

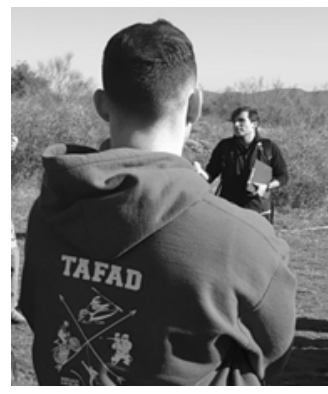

Imagen 6. Fuente: Julio Fuentesal, CES Don Bosco (Madrid),2020

\section{Ventajas de adaptar la actividad en progresión de difi- cultad.}

Permite trabajar distintos contenidos relacionados con las actividades en la naturaleza como: la cabuyería, el material técnico básico, la organización de actividades, gestión de responsabilidades y técnicas de descenso.

Posibilita la creación de una progresión en aspectos técnicos que permite detectar posibles errores antes de realizar la actividad en la práctica real, donde sería más difícil subsanarlos.

A través de las progresiones se crean niveles de dificultad dentro de la actividad, pudiendo atender a la individualidad del alumnado.

Incluso con la actividad en el centro e incluyendo módulos que simulen la cueva, se puede adaptar la actividad a poblaciones muy diversas al poder modificar el medio según las necesidades (Baena-Extremera et al., 2013; BaenaExtremera \& Granero-Gallegos, 2009).

Las instalaciones permiten realizar una progresión en los desplazamientos en distintos planos: horizontal, vertical y con desplome.

La microenseñanza permite a los discentes analizar y evaluar, dos de las categorías más altas de la taxonomía de Bloom (Krathwohl, 2002).

El grado de satisfacción y aprovechamiento en estas sesiones, hacen que la gran mayoría del alumnado la recomienden a otras promociones, haciendo que perdure el entusiasmo y se conviertan en actividades casi inmersivas recordándolas como su primer día de campamento personalizando el aprendizaje individual en cada uno de ellos.

Cómo se han recogido en puntos anteriores, seguir la metodología de Baena-Extremera (2011b) ha permitido sobrepasar los contratiempos que podría plantear la progresión en dificultad de la actividad por su naturaleza. Al plantear situaciones de aprendizaje que se asemejan al objetivo final en un entorno controlado, se prepara al discente física y mentalmente a las necesidades que va a presentar la práctica en el entorno real.

Inconvenientes adaptar la actividad en progresión de dificultad.

Requiere algún material específico y costoso que no está disponible en todos los centros y supone una inversión extra para el centro.

La responsabilidad del profesorado a la hora de plantear una actividad de esta naturaleza se eleva y sin una cobertura jurídica o un respaldo del centro, el profesor puede decidir no realizarlo por miedo a poner su responsabilidad en peligro (Escaravajal-Rodriguez \& Baena-Extremera, 2016; Santos \& Martinez, 2008

Las condiciones relacionadas con el coste económico de la actividad y el poder adquisitivo del alumnado pueden limitar el desarrollo de la actividad, como apuntan estudios anteriores (Escaravajal-Rodriguez \& Baena-Extremera, 2016; López et al., 2015; Sanjuán et al., 2020; Santos-Pastor \& Martinez-Muñoz, 2008).

Aunque un objetivo de la práctica esté centrado en la concienciación ambiental, la divulgación incontrolada de estas prácticas puede afectar de manera negativa el medio natural (Sanjuán et al., 2020).

La formación del profesorado es un factor limitante debido a la complejidad e importancia de la seguridad a lo largo de la práctica. Sabiendo que la formación de los docentes en estas prácticas es limitada, podemos considerarlo un obstáculo para el desarrollo de experiencias en el medio natural (Sáez-Padilla \& Fuentesal-García, 2014).

\section{A modo de conclusión.}

Cualquier actividad que se realice en el medio natural necesita de todo un proceso de planificación y preparación para poder cubrir los objetivos planteados y conseguir la plena satisfacción del alumnado. Algo que no podemos olvidar a la hora de poder incluir estos contenidos en nuestra programación, es la motivación (Arribas, 2008; Arufe et al., 2012). Para el discente, la novedad de materiales, vivencias y escenarios hacen que se convierta en una actividad extraordinaria, diferenciándolas de las tradicionales y clásicas.

Siguiendo el modelo propuesto, cuando desarrollamos una actividad como la espeleología y buscamos que el alumnado pueda progresar por una cueva, el profesor debe conocer y analizar todos los factores que ha de trabajar para lograr el éxito del reto propuesto. Para ello, el docente evalúa y plantea dentro del centro educativo, la forma de crear un nivel de progresión y dificultad lo más real posible para acercar al alumnado a los elementos técnicos necesarios. En este tipo de propuestas se precisa de un conocimiento previo de otras disciplinas como la cabuyería y manejo de cacharrería. Aun así, si el docente no tiene esos conocimientos, puede acudir a agentes externos que le ayuden con las exigencias técnicas de la actividad, hablamos de las Federaciones o centros especializados, como las empresas de turismo activo

Este es un intento más de aumentar y contribuir a las experiencias ya existentes, buscando alentar al profesorado a la implantación de contenidos relacionados con las actividades en el medio natural en sus planes de estudio. Sin embargo, esta es una propuesta modificable que puede ser complementada con ciertas acciones de mejora de cara a la siguiente puesta en práctica. Como autores, en línea con este objetivo consideramos que la realización de una nueva experiencia en una cueva con una orografía más compleja técni- 
camente permitiría que los discentes hiciesen una autoevaluación de ambas prácticas. Así se alcanzaría un posible incremento del valor educativo de nuestra nueva propuesta.

Somos conscientes que las dificultades en la implantación de estas actividades están presentes López et al (2015) , por eso sigue siendo necesaria más formación y más completa para dotar al profesorado de una mayor confianza para erradicar las limitaciones que llevan a ni si quiera ser planteadas (Sanjuán et al., 2020).

Finalmente, se pueden considerar nuevos objetivos y direcciones de investigación futura para seguir ampliando estos campos aún por desbrozar y abrir nuevas metodologías, nuevas metas y retos mejorando así nuestra labor como docentes y logrando un alumnado más autónomo, motivado y comprometido:

Identificar nuevos proyectos colaborativos intercentros y acciones como la compra conjunta de materiales siguiendo la línea de Sanjuán et al (2020).

Analizar y conocer la motivación del alumnado en base a los diferentes modelos de «educación aventura» para poder encontrar el método más práctico y seguro. (BaenaExtremera, 2011b).

Fomentar nuevas sinergias de participación entre entidades externas a los centros educativos como son las federaciones, clubes deportivos y empresas de turismo activo.

\section{Referencias}

AA.VV. (2007). El medio ambiente en la comunidad de Madrid. Consejeria del Medio Ambiente y Ordenación del Territorio de la Comunidad de Madrid.

Aguila, C. (2007). Las actividades físicas de aventura en la naturaleza: ¿un fenómeno moderno o posmoderno? Apunts: Educación física y deportes, 3(89), 81-87.

Aguilar, J., \& Machota, V. (2014). El buceo en la enseñanza secundaria: una propuesta práctica. Espiral. Cuadernos del Profesorado, 7(15), 70-74.

Arribas, H. F. (2008). El pensamiento y la biografía del profesorado de Actividad Física en el Medio Natural: un estudio multicaso en la formación universitaria orientado a la comprensión de modelos formativos. Universidad de Valladolid.

Arufe-Giráldez, V., Calvelo, L., González, E., \& López, C. (2012). Salidas a la naturaleza y profesorado de educación primaria. Un estudio descriptivo. EmásF: revista digital de educación física, 4(19), 30-38.

Baena-Extremera, A. (2008). La espeleología deportiva en los raids. Sportraining Magazine, 56-59.

Baena-Extremera,A. (2011b). Programas didácticos para educación física a través de la educación de aventura. Cuadernos del Profesorado, 4(7), 3-13.

Baena-Extremera, A., \& Granero-Gallegos, A. (2009). Deportes de aventura «indoor»: enseñanza de la espeleología en los institutos de educación secundaria. Tándem: Didáctica de la educación física, 30, 47-60.

Baena-Extremera, A., Serrano, J. M., Fernández, R., \& Fuentesal-Garcia, J. (2013). Adaptación de nuevos deportes de aventura a la educación física escolar: las vías ferratas. Apunts:: Educacion Fisica y Deportes, 4(114), $36-44$.

Blanco, P. C., \& Hernández-Hernández, E. (2017). Análisis de los factores universales de las actividades físicas en el medio natural / actividades físicas de aventura en la naturaleza: estudio preliminar. Espiral. Cuadernos Del Profesorado, 11(22), 61-68.

Cano, J. J., Caballero, P. J., Morenas, J., \& Parra, M. (2009). La escalada en bloque: Una propuesta de utilización de espacios clandestinos. Taìndem: Didaìctica de la Educacioìn Fiìsica, 30, 37-46.

Corrales, A. R. (2010). Trabajar la Educación ambiental desde la Educación Física To work the enviromental education from the physical education. Revista Educativa Digital.Hekademos, 5(3), 45-64.

Diamant, R. (1991). La aventura de la espeleología. Revista de educación en Ciencias Básicas e Ingeniería, 4, 1830.

Escaravajal-Rodriguez, J.-C., \& Baena-Extremera, A. (2016). La espeleología en el centro escolar, una propuesta en el área de educación física. Agora para la educacion fisica y el deporte, 18(3), 323-341.

Fernandez-Río, J. (2009). La trepa y la escalada: contenidos del bloque de actividades en el medio natural facilmente aplicables dentro del marco escolar. Apunts: Educación física y deportes, 62, 51-56.

Ferriz, R. F., González-Cutre, D., Sicilia, Á., \& Beltrán C, V. J. (2018). Estrategias motivacionales para la promoción de la actividad física en niños y adolescentes desde el contexto escolar. Inde.

Fraile, J., Gil, M., Zamorano-Sande, D., \& Sánchez-Iglesias, I. (2020). Self-regulated learning process on group work formative assessment. RELIEVE, 26(1).

Fuentesal-García, J., \& Pastor, D. (2019). El aprendizaje cooperativo y sus dimensiones. Experiencias prácticas. Educación y futuro, 21, 161-182.

García, P. J. (2002). Necesidad de regular el ejercicio y actuación de las empresas dedicadas a la prestación de servicios de turismo activo en el medio natural. Cuadernos de turismo, 9, 45-52.

Gómez-Encinas, V. (2008). Juegos y Actividades de Reto y Aventura en el contexto escolar. Wanceulen E.F. digital, 4, 1-12.

Gómez, M., \& Sanz, E. (2003). La enseñanza del esqui alpino en las clases de educación física de la educación secundaria obligatoria. RETOS. Nuevas Tendencias en Educación Física, Deporte y Recreación, 4, 11-24.

Granero-Gallegos, A. (2010). Una aproximación conceptual y taxonómica a las actividades físicas en el medio natural. Lecturas Educación Física y Deportes, Revista Digital, 12(107), 1-17.

Granero-Gallegos, A., \& Baena-Extremera, A. (2007). Importancia de los valores educativos de las actividades físicas en la naturaleza. Habilidad motriz, 29, 5-14.

Granero-Gallegos, A., Baena-Extremera, A., \& Martínez, M. (2010). Contenidos desarrollados mediante las actividades en el medio natural de las actividades de educación física en secundaría obligatoria. Agora para la educación física y el deporte, 12(3), 273-288.

Hernández, B. (2008). Los métodos de enseñanza en la Edu- 
cación Física. efdeportes.com, 132.

Krathwohl, D. R. (2002). A revision of Bloom's Taxonomy: An overview. Theory into practice, 41(4), 212-219.

León, A. N., \& Santiago, P. R. (2014). Microenseñanza una técnica para motivar el enseñar y aprender investigando. Perspectivas docentes, 52, 23-31.

López, J., Álvarez, D., \& Fernández-Miranda, S. (2015). Actividades en el medio natural acuático en educación física. Valoración del profesorado sobre los factores que limitan su inclusión. EmásF: revista digital de educación física, 33(6), 97-110.

López, S., Maneiro, R., Moral, J. E., Amatria, M., Diez, P., Barcala, R., \& Abelairas, C. (2019). Los riesgos en la práctica de actividades en la naturaleza. La accidentabilidad en la Educación Física, en las prácticas deportivas y medidas preventivas. RETOS. Nuevas Tendencias en Educación Física, Deporte y Recreación, 36(2013), 618-624.

Martínez, D., Sampedro, M., \& Veiga, O. (2007). La importancia del compromiso motor y el compromiso fisiológico durante las clases de educación física. Revista Iberoamericana de Educación, 42(2), 3.

Muñoz, A., Granado, M., Martín, J., \& Rivilla, J. (2017). Estudio de caso: Análisis de la distribución del tiempo en educación física. e-balonmano.com: Revista de Ciencias del Deporte, 13(2), 167-174.

Navarro, A. (2018). Escalada en educación primaria: Organización y aprendizajes fundamentales. EmásF: revista digital de educación física, 54(9), 60-73.

Olivera, J., \& Olivera, A. (1995). Propuesta de una clasificación taxonómica de las actividades físicas de aventura en la naturaleza: marco conceptual y análisis de los criterios elegidos. Apunts: Educación física y deportes, 41, 108-123.

Parra, M., Domínguez, G., \& Caballero, P. (2008). El cuaderno de campo: un recurso para dinamizar senderos desde la educación en valores. Agora para la educación física y el deporte, 7-8, 145-158.

Pederur. (2015). Cueva de la Bruja. Cueva de la Bruja.

Peñarrubia-Lozano, C., Guillén, R., \& Lapetra, S. (2011). Las actividades en el medio natural en Educación Física. Valoración del profesorado de Secundaria sobre los principales factores de limitación para su desarrollo. Apunts: Educación Física y Deportes, 2(104), 37-45.

Real Decreto 2048/1995, de 22 de diciembre, por el que se establece el título de Formación Profesional de Técnico superior en Animación de Actividades Físicas y Deportivas y las correspondientes enseñanzas mínimas.

Real Decreto 653/2017, de 23 de junio, por el que se establece el título de Técnico Superior en enseñanza y animación sociodeportiva y se fijan los aspectos básicos del currí- culo.

Sáez-Padilla, J. (2008). El diseño de las actividades en el medio natural en el nuevo currículo de educación secundaria obligatoria a partir de la ley orgánica de educación (LOE). Agora para la educación física y el deporte, 7-8, 99-124.

Sáez-Padilla, J., \& Fuentesal-García, J. (2014). La formacioìn universitaria en actividades físico-deportivas en el medio natural: situacioìn actual y perspectivas de futuro. Taìndem: Didaìctica de la Educacioìn Fì̀sica, 45, 1421.

Sáez-Padilla, J., Rodríguez, J. M., \& Giménez, F. J. (2011). Visión del profesorado de educación física de educación secundaria obligatoria en Andalucía sobre las actividades en el medio natural. RETOS: Nuevas Tendencias En Educación Física Como Deporte Y Recreación. 20, 915.

Sáez-Padilla, J., Tornero, I., \& Sierra, Á. (2017). Situación actual de la formación en actividades en el medio natural según el profesorado de Educación Física. Un estudio cualitativo con expertos en Andalucía. Espiral. Cuadernos Del Profesorado, 10(21), 100.

Sallent, O. (2009). El impacto ambiental de las actividades físico-deportivas en el medio natural. El caso de la práctica del Mountain Bike o bicicleta todo terreno. RETOS. Nuevas Tendencias en Educación Física, Deporte y Recreación, 2009(16), 31-35.

Sanjuán, M. F., Ricarte, M. S., \& Lozano, C. P. (2020). Trabajo colaborativo y Educación Física/ : una propuesta didáctica de escalada en Educación Primaria Collaborative work and Physical Education/ : a climbing didactic proposal in Primary Education. 37, 706-712.

Santos-Pastor, M. (2003). Las actividades en el Medio Natural en la Educación Física Escolar. Wanceullen.

Santos-Pastor, M., \& Martinez-Muñoz, L. F. (2008). Las actividades en el medio natural en la escuela. Consideraciones para un tratamiento educativo. Wanceulen E.F. digital, 4, 26-53.

Solana,A., \& Delgado, M. (2005). La aplicación práctica de la microenseñanza en la Unidad Didáctica de Deportes de Invasión. Revista de educación física: Renovar la teoría y practica, 97, 15-22.

Tejada, J. (2007). La evaluación en Educación Física en primaria en Huelva y su provincia. Universidad de Huelva.

Tejada, J., \& Sáez-Padilla, J. (2009). Educacion fisica y educacion ambiental. Posibilidades educativas de las actividades en el medio natural. Prespectivas de futuro: la educacion al aire libre y el aula naturaleza. Wanceulen E.F. digital, 5, 124-137.

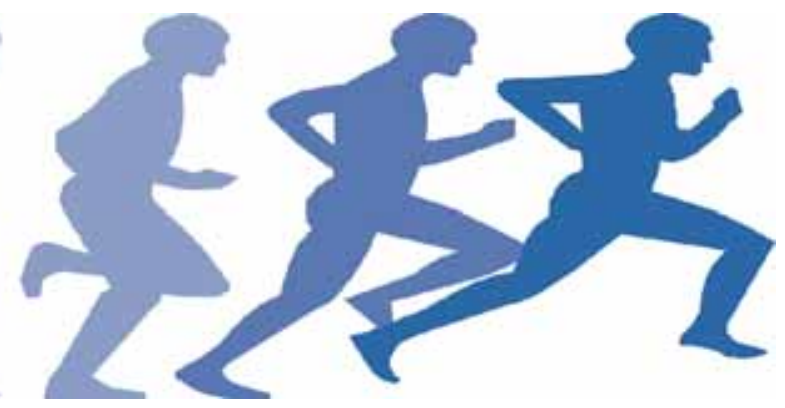

